\title{
Effect of using Propanol as internal standard on quantitative determination of ethanol in different biological matrices by head space-Gas Chromatography-Flame Ionization Detector
}

\author{
S Sudhaker ${ }^{\star}$ and Rajeev Jain ${ }^{\star \star}$ \\ IForensic Toxicology Division, Central Forensic Science Laboratory, Kolkata, India \\ ${ }^{2}$ Forensic Toxicology Division, Central Forensic Science Laboratory, Guwahati, India
}

\section{Article Info \\ Corresponding authors: \\ 1. S Sudhaker \\ Forensic Toxicology Division, \\ Central Forensic Science Laboratory, \\ Kolkata, India - 700014 \\ Email:smahatisudha@gmail.com \\ 2. Rajeev Jain \\ Forensic Toxicology Division, \\ Central Forensic Science Laboratory, \\ Guwahati, India - 781033 \\ Email: rajeevjaincfs।@gmail.com}

Received: May 23, 2016

Accepted: July 19, 2016

Published: July 22, 2016

Citation: Sudhaker S, Jain R. Effect of using Propanol as internal standard on quantitative determination of ethanol in different biological matrices by head space-Gas ChromatographyFlame Ionization Detector. Madridge J Anal Sci Instrum. 2016; 1(1): 1-3.

doi: 10.18689/mjai-1000101

Copyright: $(2016$ The Author(s). This work is licensed under a Creative Commons Attribution 4.0 International License, which permits unrestricted use, distribution, and reproduction in any medium, provided the original work is properly cited.

Published by Madridge Publishers

\begin{abstract}
Identification and quantification of ethanol in biological samples such as blood and urine is of paramount importance in forensic toxicological laboratories. Despite of the fact that there are several methods are available for quantification of ethanol in forensic samples, an accurate quantification with minimum standard deviation is still a challenging task in forensic toxicological laboratories. Headspace Gas Chromatography-Flame Ionization Detection (HS-GC-FID) is a widely used analytical method for quantitative determination of ethanol. In the present work, we evaluate effect of using propanol as internal standard in different biological metrices on ethanol quantification. Results show that using propanol as internal standard relative standard deviations are significantly reduced and calibration curves were found linear with a correlation coefficient $\left(R^{2}\right)$ of 0.999. In conclusion, HS-GC-FID method was found to be fast, accurate, sensitive and robust for quantitative determination of ethanol in complex biological matrices e.g. blood and urine.
\end{abstract}

Keywords: Headspace-Gas Chromatography-Flame Ionization Detection; Blood Ethanol Concentration; Ethanol Quantification; Forensic Toxicology; Propanol.

\section{Introduction}

Ethanol is the most socially-accepted psychoactive drug over the globe which is generally accepted and legal in many countries. Ethanol can have life-threatening health hazards. Its pleasures are widely acknowledged and form a bond of community for the majority of adults in Western countries. However, because of its antidepressant nature, ethanol can affect one's motor vehicle driving capacity [1]. Hence, measurement of blood-ethanol concentration (BEC) becomes crucial to access whether a person driving under the influence of ethanol. Additionally, quantitative analysis of ethanol in biological samples can aid in determining manner and cause of death [2]. Beside this, BEC is also needed in situations like drug-facilitated sexual assaults (DFSA), workplace drug monitoring and post-mortem analysis [3].

The most common method of $\mathrm{BEC}$ determination is breath analysis to check whether a suspected person is driving under the influence of ethanol. Owing to its non-invasive nature of analysis breath analysis became widely used practice among law enforcement agencies. There is a correlation between the concentration of ethanol in breath and concentration of ethanol in blood. The amount of ethanol present in $2100 \mathrm{~mL}$ of breath 
is equal to amount of ethanol present in $1 \mathrm{~mL}$ of blood at constant temperature i.e. $34^{\circ} \mathrm{C}$. However, the error reported in analysis of ethanol in breath is as high as $\pm 50 \%[3,4]$.

Acute ethanol toxicity is commonly encountered in the clinical \& forensic cases. Majority of fatal ethanol intoxication cases are associated with consumption of alcoholic beverages. The most commonly used specimen other than breath for ethanol determination is blood. Urine is also used occasionally followed by saliva.

Attempts have been made to simplify the methods available for the determination of ethanol and to develop new strategies that can be used with low cost instrumentation and validated method without problems. The methods available for determination of ethanol in literature are based on physical measurements which are carried out after previous distillation of the sample in order to separate the ethanol. Various chemical methods are available for determination of ethanol in tissue and body fluids based on oxidation of ethanol to acetic acid with potassium dichromate and the determination of the excess oxidizing agent using thiosulphate [5]. In recent times, techniques like enzymatic determination of ethanol have gained a lot of attention. IR spectrometry in mid IR and near IR is an interesting alternative to directly determine the quantity of alcohol in alcoholic beverages [6]. The high absorbance of IR radiation by ethanol and water has been reduced by employing cells of appropriate material with small thickness [7]. However, ethanol can be determined in presence of water in Near IR region using common glass without any need of short path lengths [6].

The choice of selection of stationary phases in GC for separation of volatile compounds such as ethanol, methanol, n-propanol, isopropanol etc provides a sensitive and selective analytical method which is immensely crucial and significant in court of law. The aim of present study was to identify the effect of using propanol as internal standard on the quantitative determination of ethanol by headspace-gas chromatography-flame ionization detector (GC-FID).

\section{Chemicals and reagents}

All chemicals and reagents used in this study were of analytical grade, unless otherwise stated. Ethanol, propanol, tartaric acid and distilled water were procured from Merck (Dermstadt, Germany). Round bottomed flask, water condenser, long tube, measuring jar and a test tube of certified good quality glass wares were used.

\section{Headspace-Gas Chromatography-Flame Ionization Detection (HS-GC-FID) Conditions}

The HS-GC-FID used in this study was Perkin Elmer Clarus 600 gas chromatograph. The GC was equipped with PoraPak-Q capillary column having ethylvinylbenzene-divinylbenzene (EVB-DVB) stationary phase (30 m length, $0.125 \mathrm{~mm}$ I.D.). Helium (99.99\%) was used as carrier gas at a flow rate of $1 \mathrm{~mL} / \mathrm{min}$. Oven temperature was isothermal which was kept at $160^{\circ} \mathrm{C}$ throughout the run. Injector port and detector temperature were kept at
$20{ }^{\circ} \mathrm{C}$, respectively. One $\mu \mathrm{L}$ of distillate was subjected to HS-GCFID analysis. The retention time $\left(T_{R}\right)$ and peak area of samples were recorded and matched with those of standard ethanol to confirm the presence of ethanol. HS-GC-FID chromatogram is shown in figure 1. $T_{R}$ of ethanol and propanol was 1.992 and $2.452 \mathrm{~min}$, respectively. For additional identification, the sample was also analyzed on GC-MS system and the mass spectra of ethanol and its NIST library match are shown in figure 2.

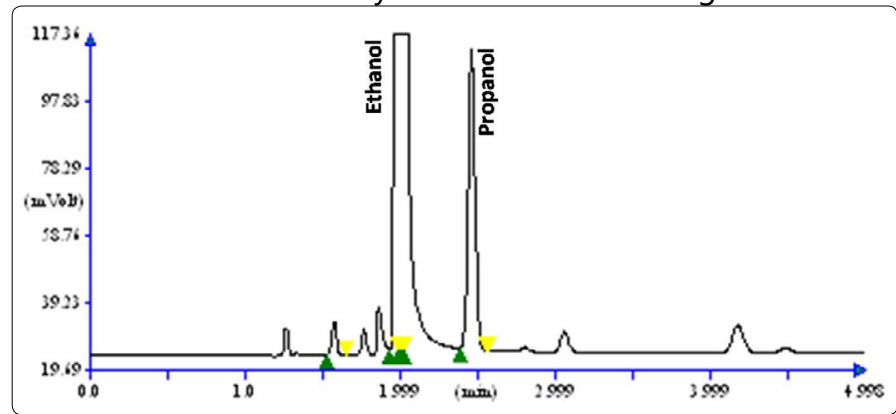

Figure 1: HS-GC-FID chromatogram of ethanol along with propanol as internal standard in blood sample.

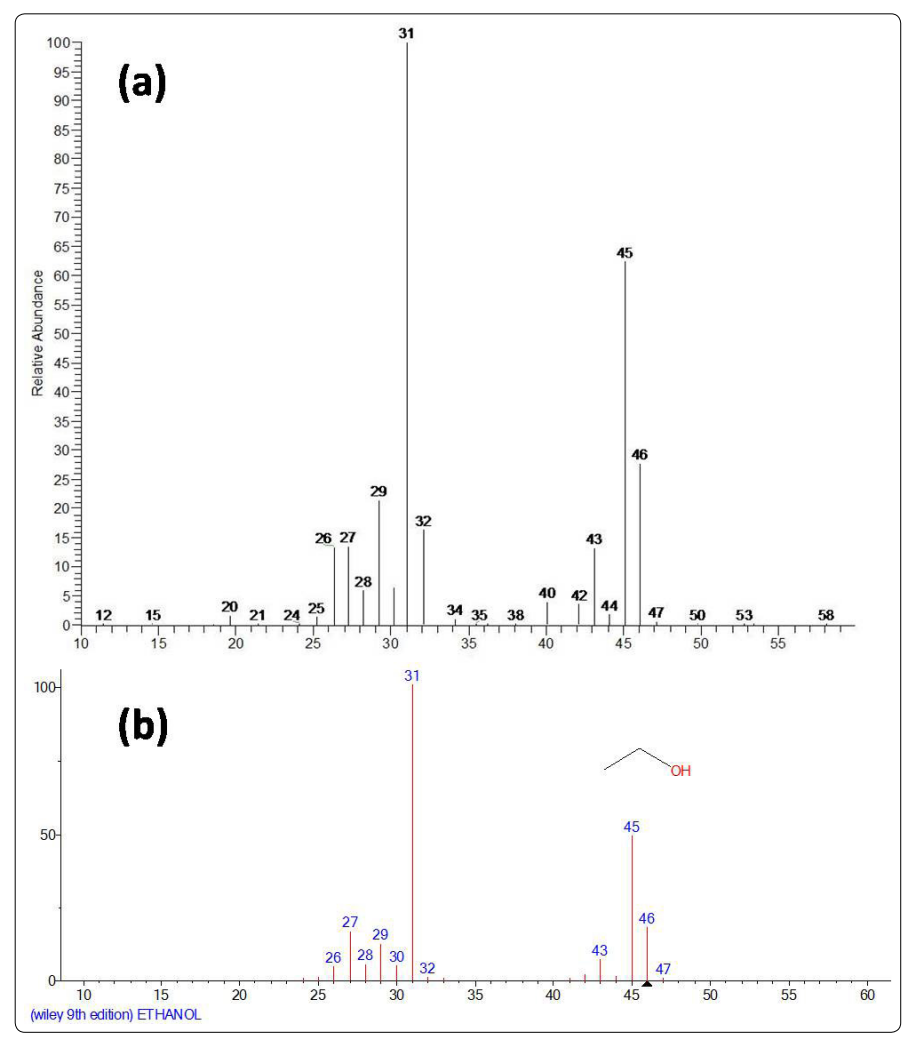

Figure 2: (a) Mass spectra of ethanol in sample (b) NIST library match of mass spectra of ethanol

\section{Sample preparation}

One $\mathrm{mL}$ of sample (blood or urine) was taken in a round bottom flask. To this, $5 \mathrm{~mL}$ of tartaric acid was added as deproteinizing agent followed by addition of $10 \mathrm{~mL}$ of distilled water. The mixture was then shaken well and distillated by reflux condenser for $15 \mathrm{~min}$. After the distillation process is over, about 3-5 mL of distillate was collected. This distillate was used for preliminary color tests such as lodoform test and Dichromate test; to confirm the presence of ethanol. Final quantification was performed using HS-GC-FID. 


\section{Method validation parameters}

Under optimized conditions of HS-GC-FID, blank blood and urine samples were spiked with known amount of ethanol to study and a calibration graph was plotted between blood concentration and peak area ratio (ratio of peak area of ethanol and propanol) in the range of $0.02-0.2 \mathrm{mg} \%$ (figure 2). The intra and inter day precision (repeatability and reproducibility) were studied at three different concentration levels of calibration graph (low, middle and high) and was expressed as percent relative standard deviation (\%RSD). Absolute recoveries of ethanol were also calculated at low, middle and high concentration levels of calibration graph. Limit of detection (LOD) and limit of quantification (LOQ) were calculated at a signal-to-noise ratio of $3: 1$ and 10:1, respectively.

\section{Results and discussion}

Ethanol was detected in the samples of Blood and urine. The sample $T_{R}$ was matched with the $T_{R}$ of standard ethanol. GC is routinely used in forensic labs for the detection of ethanol in blood and urine samples. Before applying it to real sample, the method was thoroughly optimised and validated for its linearity, LOD, LOQ precision and recovery. A good linearity was observed in the calibration range of $0.02-0.2 \mathrm{mg} \%$ of ethanol with a square of correlation coefficient (R2) of 0.998 and 0.999 in blood and urine samples, respectively. Intra and inter-day precisions $(n=5)$ expressed as \% RSD were studied for ethanol in spiked blood and urine samples and were found to be in the range of 1.83$6.57 \%$, with propanol. On the other hand, intra and inter-day precisions without propanol were found to be little higher (3.17$8.37 \%$ ) which shows that there is still significant matrix effect on ethanol quantification. Recovery assay of ethanol were performed directly in spiked blood samples and were found to be in the range of $81-94 \%$ (Table 1). From table 1, it is also evident that recovery of ethanol increased with increasing concentration in blood and urine. Similarly, the recovery of ethanol in urine is significantly higher in than blood. This can be explained by the fact that, at lower concentrations, recovery is less due to higher matrix effect and as the concentration increases the recoverable amount of analyte also increased which subsequently increases the overall recovery. In comparison to urine, obviously, blood is more complex biological matrix therefore; the recovery of ethanol in blood was lesser than urine sample.

\begin{tabular}{|c|c|c|c|c|c|c|c|c|c|c|}
\hline \multirow[t]{2}{*}{ Matrix } & \multirow{2}{*}{$\begin{array}{l}\text { Linearity } \\
(\mathrm{mg} \%)\end{array}$} & \multirow{2}{*}{$\begin{array}{l}\text { LOD } \\
(\mathrm{mg} \\
\%)\end{array}$} & \multirow{2}{*}{$\begin{array}{c}\text { LOQ } \\
\text { (mg } \\
\%)\end{array}$} & \multicolumn{3}{|c|}{$\begin{array}{l}\text { Precision with POH / } \\
\text { Without POH (mg \%) }\end{array}$} & \multirow[t]{2}{*}{$\mathbf{R}^{2}$} & \multicolumn{3}{|c|}{$\begin{array}{c}\text { Recovery/ \%RSD } \\
\text { (mg \%) }\end{array}$} \\
\hline & & & & 0.02 & 0.1 & 2 & & 0.02 & 0.1 & 2 \\
\hline Blood & $0.02-2$ & 0.005 & 0.017 & $6.57 / 8.37$ & $4.13 / 6.12$ & $2.98 / 4.64$ & 0.998 & $81 / 4.7$ & $84 / 4.2$ & $86 / 3.8$ \\
\hline Urine & $0.02-2$ & 0.006 & 0.019 & $5.89 / 7.56$ & $3.69 / 5.83$ & $1.83 / 3.17$ & 0.999 & $85 / 4.1$ & |90/3.8 & $394 / 3.5$ \\
\hline
\end{tabular}

Table 1: Method validation parameters of ethanol in spiked blood and urine sample

Results show that there was a significant effect of using propanol as an internal standard on the repeatability of the method. The intra and inter day precision of widely used HSGC-FID method has been reduced to 1.81 and $1.71 \%$, respectively by using propanol as internal standard. The method has been successfully applied for the quantification of ethanol in real blood and urine samples. The samples were prepared following the standard protocol and analyzed by HS-GC-FID method. Concentration of ethanol in blood and urine was found to be 0.16 and $0.14 \mathrm{mg} \%$ respectively.

\section{Conclusion}

Blood and urine examination helps the investigators to know the presence or absence of ethanol and plays an important role in the criminal investigation. Hence it is needed to analyse and estimate the amount of ethanol in blood and urine samples in the suspected viscera samples in Medico legal cases up to sub mg level. The method is sensitive and easy. In this study a number of blood and urine samples have been analysed suspected to be the presence of ethanol routinely encountered as case exhibits in the laboratory. All the suspected exhibits are analysed with available methods. Detection and estimation of ethanol was achieved by GC method with external and internal standard method. Since the analytical method is fully automated, the manual error in the sampling and injection is reduced by using an HS auto sampler and internal standard. The evaluated internal standard method will be useful for the quantitative determination of ethanol. Matrix effect and relative standard deviations are significantly minimized by using propanol as internal standard for quantitative determination of ethanol in blood and urine samples. Therefore, this method can be routinely used for the said purpose in various forensic toxicological laboratories.

\section{References}

1. Cowan DM, Maskrey JR, Fung ES et al. Best-practices approach to determination of blood alcohol concentration (BAC) at specific time points: Combination of ante-mortem alcohol pharmacokinetic modelling and postmortem alcohol generation and transport consideration. Regul. Toxicol. Pharmacol. 2016; 78: 24-36. doi: 10.1016/j.yrtph.2016.03.020. Epub 2016 Apr 1

2. Saitman A, Estrada J, Fitzgerald RL, Mcintyre IM. Comparative analysis of hospital and forensic laboratory ethanol concentration: A 15 month investigation of antemortem specimens. J. Forensic Leg. Med. 2015; 33: 23-27. doi: 10.1016/j.jflm.2015.03.012.

3. Kelly AT, Mozayani A. An overview of alcohol testing and interpretation in the 21st century. J. Pharm. Pract. 2012; 25(I): 30-36. doi: $10.1177 / 0897190011431149$.

4. Krikku P, Wilhelm L, Jenckel S et al. Comparison of breath-alcohol screening test results with venous blood alcohol concentration in suspected drunken drivers. Forensic Sci. Int. 2014; 239: 57-6. doi: 10.1016/j.forsciint.2014.03.019

5. Winnick T. Determination of ethyl alcohol by microdiffusion. Ind. Eng Chem. Anal. Ed. 1942; 14(6): 523-525. doi: 10.1021/i560106a029

6. Gallignani M, Garrigues S, Guardia MDL. Direct determination of ethanol in all types of alcoholic beverages by near-infrared derivative spectrometry. Analyst. 1993; 118: 1167-1173. doi: 10.1039/AN9931801167

7. Mahia PL, Gandara JS, Losada PP. Validation of ethanol determination in alcoholic beverages by infrared spectrophotometry using orthogonal and derivative functions to correct for water absorption. Vib. Spectrosc. 1992; 3(2): 133-138. doi: 10.1016/0924-2031(92)80006-N 